\title{
Quantitative Analysis of Elemental Depth on Wendelstein 7-X Divertor Baffle Screws by Picosecond Laser-Induced Breakdown Spectroscopy
}

\author{
Cong Li ${ }^{1,2}$, Jannis Oelmann², Sebastijan Brezinsek ${ }^{2}$, Marcin Rasinski ${ }^{2}$, Chandra Prakash Dhard ${ }^{3}$, Ralf \\ König $^{3}$, Yunfeng Liang ${ }^{2}$, Hongbin Ding ${ }^{1, *}$, Christian Linsmeier ${ }^{2}$ and the W7-X team ${ }^{3}$ \\ ${ }^{1}$ Key Laboratory of Materials Modification by Laser, Ion and Electron Beams (Ministry of Education), School of \\ Physics, Dalian University of Technology, 116024 Dalian, P. R. China \\ ${ }^{2}$ Forschungszentrum Jülich GmbH, Institut für Energie- und Klimaforschung - Plasmaphysik, Partner of the \\ Trilateral Euregio Cluster (TEC), 52425 Jülich, Germany \\ ${ }^{3}$ Max-Planck-Institut für Plasmaphysik, 17491 Greifswald, Germany \\ *Corresponding authors: E-mail: hding@dlut.edu.cn (H. Ding)
}

\begin{abstract}
:
Analysis of elemental distributions in plasma-facing components (PFCs) is vital for the study of material erosion, deposition, and fuel retention in Wendelstein 7-X (W7-X) stellarator operating in full 3D geometry. In this work, we report the results on the application of picosecond laser-induced breakdown spectroscopy (ps-LIBS) combined with laser-induced ablation quadrupole mass spectrometry (LIA-QMS) to quantitatively investigate the elemental depth distribution and fuel retention on the screws PFCs from the divertor baffle of W7-X. Depth profiles of $\mathrm{H}, \mathrm{C}, \mathrm{Ti}$ and Mo intensities on the screws with specific multilayer structures have been achieved by LIBS combined with LIA-QMS. A linear correlation approach has been applied for identifying the interface between the C layer and the Ti layer and shows that a laser ablation rate of $(82.9 \pm 0.9) \mathrm{nm} / \mathrm{shot}$ can be achieved on $\mathrm{C}$ layer thickness measurement by a laser energy density of $6.5 \mathrm{~J} / \mathrm{cm}^{2}$. Moreover, quantitative $\mathrm{H}$ retention content on the surface of the screws with stellarator discharge plasma exposure is approximately $1.1 \times$ $10^{21}$ atoms $/ \mathrm{m}^{2}$ which is higher than approximately $3.5 \times 10^{20}$ atoms $/ \mathrm{m}^{2}$ on the surface of the new screw without plasma exposure. The studies well demonstrate the ability to determine elementary resolved layer thicknesses and $\mathrm{H}$ retention measurements by depth analysis of LIBS on PFCs exposed in W7-X.
\end{abstract}

Keywords: laser-induced breakdown spectroscopy; plasma wall interaction; deposition layer; fuel retention 


\section{Introduction}

Wendelstein 7-X (W7-X) [1] is the largest optimized stellarator in the world and has been optimized with respect to good magneto-hydrodynamics (MHD) stability, improved neoclassical confinement, good confinement of fast particles, and low bootstrap current [2]. It is designed for a steady-state plasma operation and provides a unique platform to study the 3D plasma-wall interaction (PWI) processes [3,4]. These studies include material erosion, transport, deposition and mixing (full material migration cycle) as well as fuel retention by implantation and co-deposition in and on plasma-facing components (PFCs) such as divertor target plates, heat shields and baffles.

In order to map the erosion/deposition pattern, amorphous carbon (a-C) coated Ti-Zr-Mo (TZM) screws have been installed in baffle region of a divertor module of W7-X. The screws have a multilayer structure with a certain thickness of $\mathrm{C}$ layer on the top surface to protect the plasma from potential metallic influx due to the used TZM material. The $\mathrm{C}$ layer might be thinner and represent the residual layer in the case the screw was in a net erosion zone or it might be thicker and an additional C layer by plasma deposition is on the top of the artificial protection layer. This structure provides a unique sample to investigate the PWI issues, such as erosion, deposition and fuel retention. To study those, an elemental analysis method on the PFCs with spatial and depth resolution is essential. Several post-mortem methods, such as nuclear reaction analysis (NRA) [5], secondary ion mass spectroscopy (SIMS) [6], thermal desorption spectrometry (TDS) [7] etc., have been used for the ex situ material analysis for PWI study. However, post-mortem analysis cannot provide the real-time elemental information of PFCs, especially during the long-pulse discharge operation. It will be more difficult or even inhibited in the future fusion device due to the actively cooled structures of PFCs [8].

Laser-induced breakdown spectroscopy (LIBS) is a well-established elemental analysis approach $[9,10]$. Its excellent capability has been well demonstrated not only in the lab [11-16] but also in fusion devices such as EAST [17] and TEXTOR [18] for in situ studies. Graphite limiter tiles from the initial operational phase in limiter configuration (OP1.1) of W7-X has been carried out by post-mortem analysis of LIBS. The erosion zone and the deposition zone has been measured along the toroidal direction of the limiter. It shows that the $\mathrm{H}$ retention content in the deposition dominated zone is higher than in the other zones $[19,20]$. A LIBS system, as a part of a dedicated set of diagnostic systems for PWI study in W7-X [21], is currently under design for in situ and real-time diagnostic of the codeposition and fuel retention. However, here we focus on the ex situ studies as no LIBS system is yet installed in W7-X. Currently, the passively cooled graphite divertor has been used in W7-X since OP1.2a campaign in 2017 [1]. The $\mathrm{C}$ deposition is accompanied by co-deposition with the plasma fuel

$-\mathrm{H}$ in this case [22]. Therefore, the elemental depth analysis, especially for $\mathrm{C}$ and $\mathrm{H}$, is very essential 
for the PWI study in W7-X. Although it is still very challenging to achieve the quantitative $\mathrm{H}$ content by only LIBS methods, LIBS combined with laser-induced ablation quadrupole mass spectrometry (LIA-QMS) [20,23] which is one of a limited number of post mortem analysis diagnostics which allows quantitative and depth-resolved elemental information can provide additional information for the quantitative $\mathrm{H}$ content.

In this work, LIBS combined with LIA-QMS with picosecond (ps) pulse laser is employed to investigate the screws from the baffle region of a divertor module of W7-X for PWI research. The elemental depth profiles are achieved by both LIBS and LIA-QMS. The linear correlation approach is applied to improve the elemental depth profiles and identify the interface between two layers. The LIBS ablation rate is achieved by using the correlation between the LIBS result and the accurate C layer thickness. In addition, quantitative contents of $\mathrm{H}$ retention on the surface of the new screws and exposed screws are also investigated.

\section{Materials and methods}

\subsection{Screw samples}

The screws PFCs were widely installed in the W7-X plasma vessel to fix the graphite tiles for divertor baffles, heat shield and toroidal closures in OP1.2a [24]. The screws have a specific multilayer structure on the TZM substrate which is shown in fig. 1. The amorphous $C$ coated protective layer with different thicknesses on the top surface of the screw was made by magnetron sputtering. This layer was used to protect the metal sputtering from the substrate of the screw and avoid metal impurity production during the discharge plasma exposure, because the graphite divertors have been used in W7-X since OP1.2a campaign. Between the $\mathrm{C}$ layer and the TZM substrate, a Ti layer with a thickness of approximately $1 \mu \mathrm{m}$ was embedded. In this work, four new screws with $\mathrm{C}$ layer thickness between 5.8 $\mu \mathrm{m}$ to $12.0 \mu \mathrm{m}$ without plasma exposure, and two exposed screws named \#14 and \#34 which were exposed to about $60 \mathrm{~min}$ of $\mathrm{H}$ and helium (He) plasma operation in $\mathrm{W} 7-\mathrm{X}$ were investigated. The locations of the \#14 screw and \#34 screw were at the inboard side baffle and outboard side baffle of lower divertor, respectively. The accurate thickness of $\mathrm{C}$ layer can be clearly determined by crosssectional scanning electron microscopy (SEM) image and focused ion beam (FIB) cutting on the top surface of the screws. The $\mathrm{C}$ layer thicknesses of the four new screws are $5.83 \mu \mathrm{m}, 6.96 \mu \mathrm{m}, 9.31 \mu \mathrm{m}$ and $11.25 \mu \mathrm{m}$, respectively. The C layer thicknesses of \#14 screw and \#34 screw are $11.81 \mu \mathrm{m}$ and 7.75 $\mu \mathrm{m}$, respectively. 


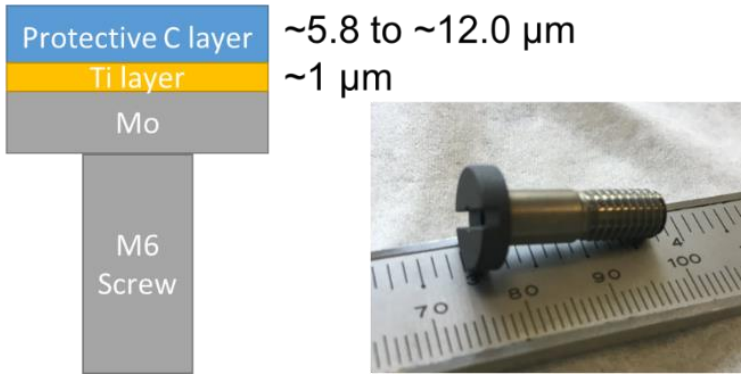

Fig. 1. Schematic and photo of the W7-X screws. The substrate of the M6 screw was TZM. Protective C layer with a thickness of approximately $5.8 \mu \mathrm{m}$ to $12.0 \mu \mathrm{m}$ on the top surface of the screws.

\subsection{Laboratory setup}

The detail of the LIBS and LIA-QMS system can be found in our previous works $[19,20]$. A Qswitched laser (PL2241, EKSPLA) at a third harmonic wavelength of $355 \mathrm{~nm}$ with a pulse length of $t_{p}$ $=35 \mathrm{ps}$ was used to ablate the target and produce the LIBS plasma in a vacuum chamber with a background pressure of $10^{-5} \mathrm{~Pa}$. The short pulse duration of this setup in ps decreases the heat penetration depth $L_{H P D}=\sqrt{2 D t_{p}}$ with thermal diffusivity $D$ by several orders of magnitude [25]. The laser energy density of $6.5 \mathrm{~J} / \mathrm{cm}^{2}$ was achieved by using the laser energy of $25 \mathrm{~mJ} / \mathrm{shot}$ with laser spot size of $0.7 \mathrm{~mm}$ diameter. In contrast to our previous experiments, besides an overview spectrometer with a wavelength from $350 \mathrm{~nm}$ to $800 \mathrm{~nm}$ (HR2000, Ocean Optics), a high-resolution spectrometer in Littrow arrangement with a grating of 1200 lines/mm and a wavelength range of $15 \mathrm{~nm}$ equipped an ICCD camera (iStar 334, Andor) was also used. The light collection angle was set to $80^{\circ}$ or $45^{\circ}$ to the surface of the sample. The LIA-QMS method was used simultaneously with LIBS to quantify the $\mathrm{H}$ retention result.

\section{Results and discussion}

\subsection{LIBS spectra}

The LIBS spectra are recorded for successive laser shots at the same position on the surface of each screw to obtain the depth profile analysis. The 5th, 120th and 250th spectra are the typical LIBS spectra from the $\mathrm{C}$ layer, Ti layer and TZM substrate in the new screw with $\mathrm{C}$ layer thickness of $9.31 \mu \mathrm{m}$ and shown in fig. 2. The $\mathrm{H}$ and $\mathrm{C}$ spectral peaks are found from LIBS spectrum in the $\mathrm{C}$ layer. Because the energy profile of laser is Gaussian and not perfectly rectangular, the Ti signal always accompanies by the $\mathrm{C}$ signal and Mo signal in the LIBS spectrum from the Ti layer. Strong Mo signal is found from the LIBS spectrum in the TZM substrate. The He content on the screw is lower than the limit of detection of the LIBS system, because of very few He retention in the deposition layer. 


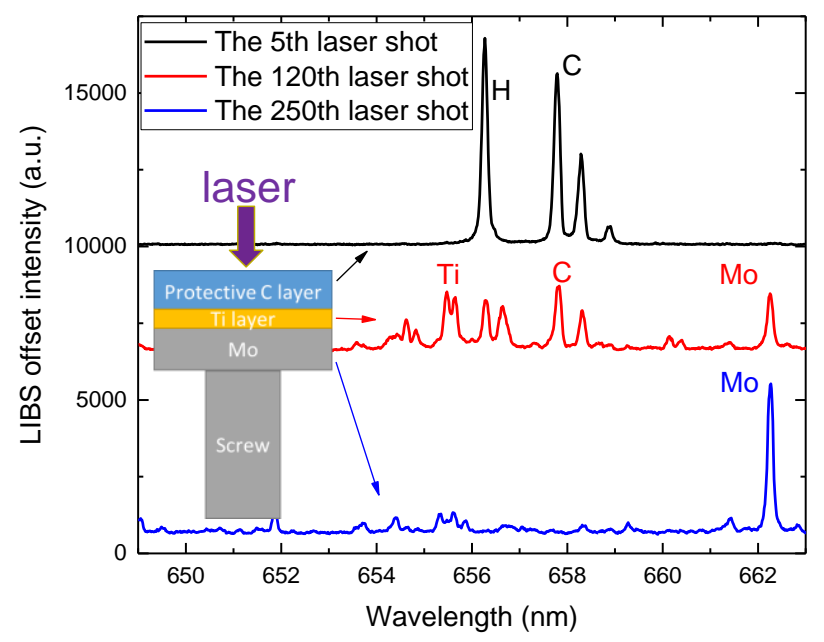

Fig. 2. Typical LIBS spectra with the different number of laser shots to show the characteristic spectral peaks in $\mathrm{C}$ layer, Ti layer and Mo substrate on a new screw (C layer thickness of $9.31 \mu \mathrm{m})$.

\subsection{LIBS and LIA-QMS elemental depth profile}

Elemental depth profiles results can provide the erosion and deposition information about the PFCs. The typical LIBS elemental depth profiles of Ti I (655.6 nm), H I (656.3 nm), C II (657.8 nm) and Mo $\mathrm{I}(661.9 \mathrm{~nm})$ on the new screw with $\mathrm{C}$ thickness of $9.31 \mu \mathrm{m}$ are shown in fig. 3. The $\mathrm{H}$ signal intensity is very high at the top surface of the new screw due to the adsorption of the water from the atmosphere. Subsequently, the $\mathrm{H}$ and $\mathrm{C}$ intensities behaviors are similar and decrease with depth, thus the $\mathrm{H}$ was embedded in the $\mathrm{C}$ layer during the deposition process of the layer induced by magnetron sputtering. The Ti layer is the interface layer between the $\mathrm{C}$ layer and the Mo substrate, so the Ti intensity increases and then decreases with depth. When the Ti intensity reaches the maximum value, there are also $\mathrm{C}$ and Mo signals due to the influence of the energy profile of laser. The Mo intensity from the substrate increases and then plateaus with the depth. 


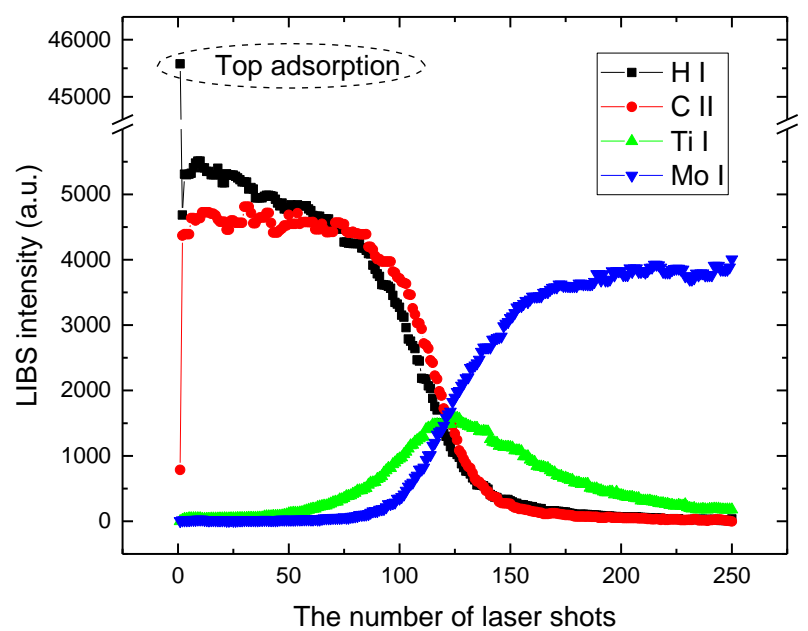

Fig. 3. LIBS elemental depth profiles of Ti I $(655.6 \mathrm{~nm})$, H I $(656.3 \mathrm{~nm})$, C II $(657.8 \mathrm{~nm})$ and Mo I $(661.9 \mathrm{~nm})$ intensities on the new screw with $\mathrm{C}$ thickness of $9.31 \mu \mathrm{m}$.

During the LIBS measurement, the $\mathrm{m} / \mathrm{z}$ from the laser ablated residual gas was analyzed simultaneously by the LIA-QMS method. The comparison of depth profiles of normalized C signal by LIBS and LIA-QMS on the screws with C thickness of $11.81 \mu \mathrm{m}$ and $7.75 \mu \mathrm{m}$ is shown in fig. 4 . The LIBS result is from the C II emission intensity at the wavelength of $657.8 \mathrm{~nm}$. The LIA-QMS result is from the signal intensity of $\mathrm{m} / \mathrm{z}$ of $12 \mathrm{u} / \mathrm{e}$. The $\mathrm{C}$ signal of the quadrupole was form the hydrocarbon gases such as $\mathrm{CH}_{4}$ and $\mathrm{C}_{2} \mathrm{H}_{6}$ which were produced by laser ablation on $\mathrm{C}$ material. Both LIBS and LIAQMS intensities decrease after tens of laser shot when the laser ablation depth reaches the Ti layer. 


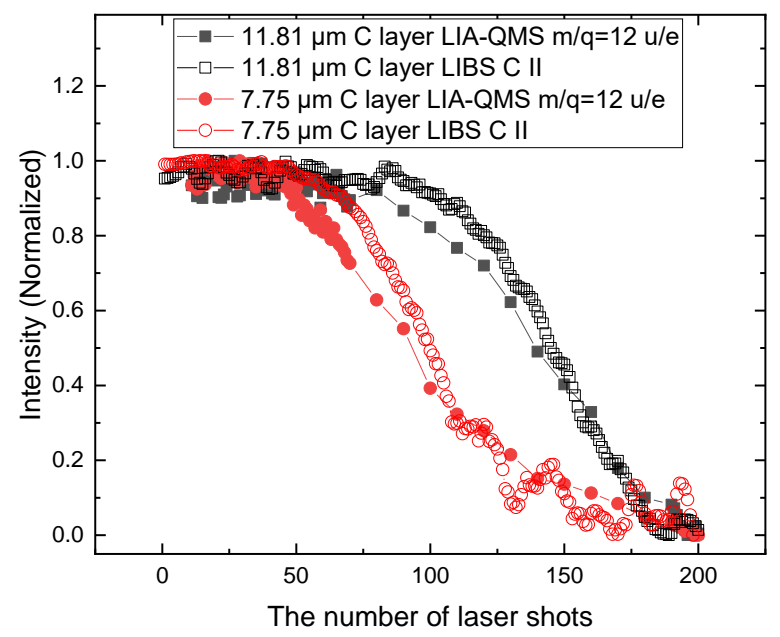

Fig. 4. The comparison of depth profiles of LIBS C II $(657.8 \mathrm{~nm})$ and LIA-QMS $(\mathrm{m} / z=12 \mathrm{u} / \mathrm{e})$ on the screws with $\mathrm{C}$ thickness of $11.81 \mu \mathrm{m}$ and $7.75 \mu \mathrm{m}$.

\subsection{C layer thickness measurement by LIBS}

The measurement and determination of $\mathrm{C}$ layer thickness on screws by LIBS are important goals of this work. Thus, the local erosion/deposition balance can be studied here in a kind of marker layer approach. It will be very useful for the on-line and real-time analysis of erosion and deposition on PFCs in the fusion device by an in situ LIBS system. The distinction of $\mathrm{C}$ layer and Ti layer is very important to measure the thickness information about the $\mathrm{C}$ layer. However, due to the Gaussian laser energy profile, it is difficult to accurately determine the interface between $\mathrm{C}$ layer and Ti layer in the screw only by using the elemental intensity profiles which are shown in fig. 3 .

Here, a linear correlation approach $[26,27]$ is carried out to obtain the LIBS depth profiles and distinguish the different layers. The linear correlation measures the degree of interrelation between two variables, $x$ and $y$, through the linear correlation coefficient, $r$, calculated using the following formula:

$$
r=\frac{\sum_{i}\left(x_{i}-\bar{x}\right)\left(y_{i}-\bar{y}\right)}{\sqrt{\sum_{i}\left(x_{i}-\bar{x}\right)^{2}} \sqrt{\sum_{i}\left(y_{i}-\bar{y}\right)^{2}}}
$$

where $x$ and $y$ are two data sets, $\bar{x}$ is the mean of all $x_{i}$, and $\bar{y}$ is the mean of all $y_{i}$. The value of $r$ lies between -1 and +1 , which can be used to indicate the correlation intensity between $x$ and $y$. An $r$-value with near to 1 corresponds to complete positive correlation, when $x$ and $y$ spectra are from the very similar elements. If the $r$-value is near to zero, then the $x$ and $y$ are uncorrelated. 
Depth profiles with linear correlation are finally obtained by plotting the evolution of the linear correlation coefficient value for the three reference spectra from the $\mathrm{C}$ layer, the Ti layer and the Mo substrate along the depth sequence of spectra. The spectral regions between $649 \mathrm{~nm}$ and $663 \mathrm{~nm}$ are selected for the calculation of the linear correlation coefficient. The reference spectra are obtained independently for each screw. The reference spectrum for the $\mathrm{C}$ layer is the average spectrum from the 3rd to the 7th depth sequence spectra to avoid the influence of $\mathrm{H}$ intensity on the surface. The reference spectrum for the Ti layer is the average spectrum from five spectra around the spectrum which has the maximum Ti LIBS intensity. The reference spectrum for the Mo substrate is the average spectrum from the 246th to the 250th depth sequence spectra. Then, the linear correlated depth profiles are shown in fig. 5. On the first 1 or 2 laser shots, the correlation coefficients for the $\mathrm{C}$ layer are low because the very high $\mathrm{H}$ intensities are obtained on the surface of the screws. Then, from the third spectrum, the correlation coefficients for the $\mathrm{C}$ layer are very near to 1 which indicates that the LIBS spectra are from the $\mathrm{C}$ layer. Then, with the depth increasing, the correlation coefficients for the $\mathrm{C}$ layer decreases and the correlation coefficients for the Ti layer increases. The crossing point means that the spectra from this depth are more correlated to the Ti layer than to the $\mathrm{C}$ layer. So the interface between the $\mathrm{C}$ layer and the Ti layer can be clearly identified and shown as dotted lines in fig. 5 . 


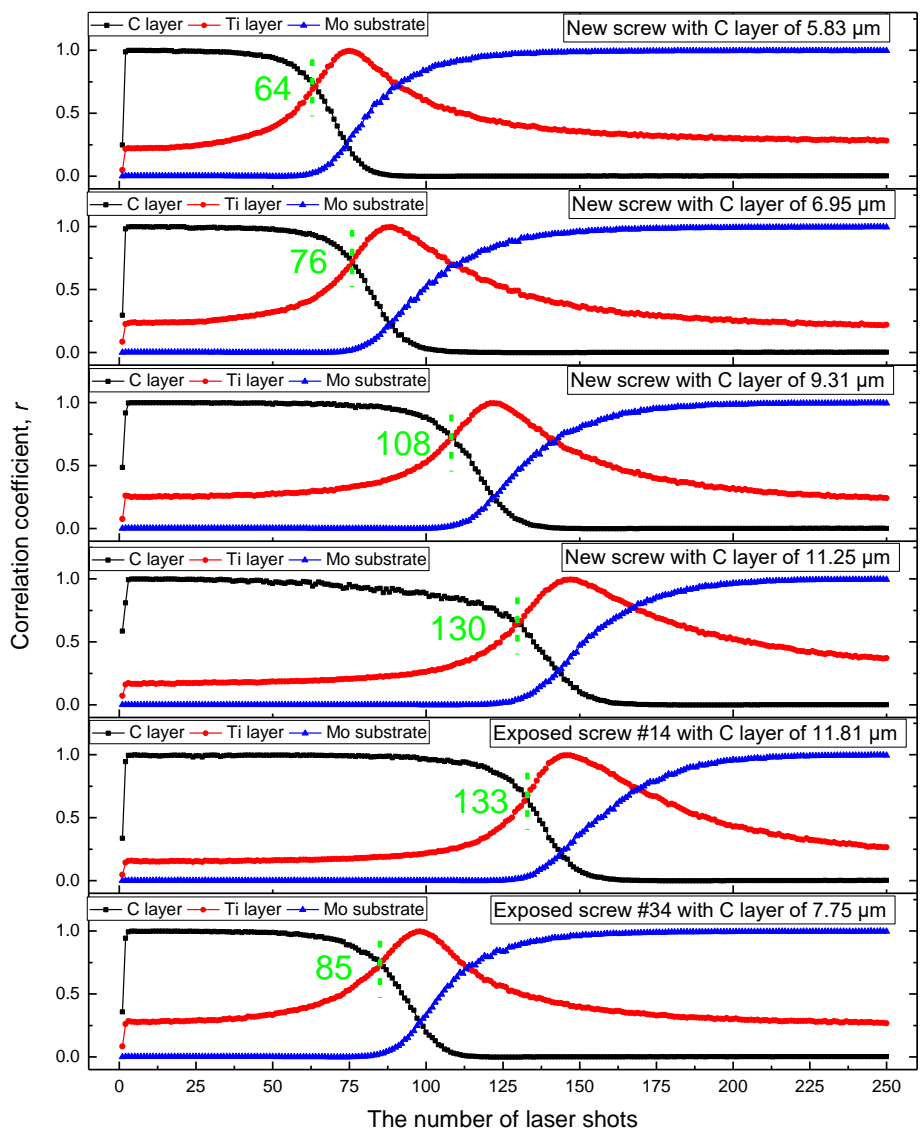

Fig. 5. Correlated depth profiles for the $\mathrm{C}$ layer, the Ti layer and the Mo substrate on four new screws and two exposed screws. The dotted lines show the location of the interface between the $\mathrm{C}$ layer and the Ti layer.

The correlation between the number of laser shot reaching on the interface between the $\mathrm{C}$ layer and the Ti layer and the $\mathrm{C}$ layer thickness which is measured by FIB cutting is shown in fig. 6 . The results can be well linearly fitted and show that LIBS ablation rate is accurately calibrated. The ablation rate of $(82.9 \pm 0.9) \mathrm{nm} /$ shot is achieved by a laser energy density of $6.5 \mathrm{~J} / \mathrm{cm}^{2}$. These indicate that the LIBS depth analysis with linear correlation approach can accurately measure the $\mathrm{C}$ thickness on the PFCs. 


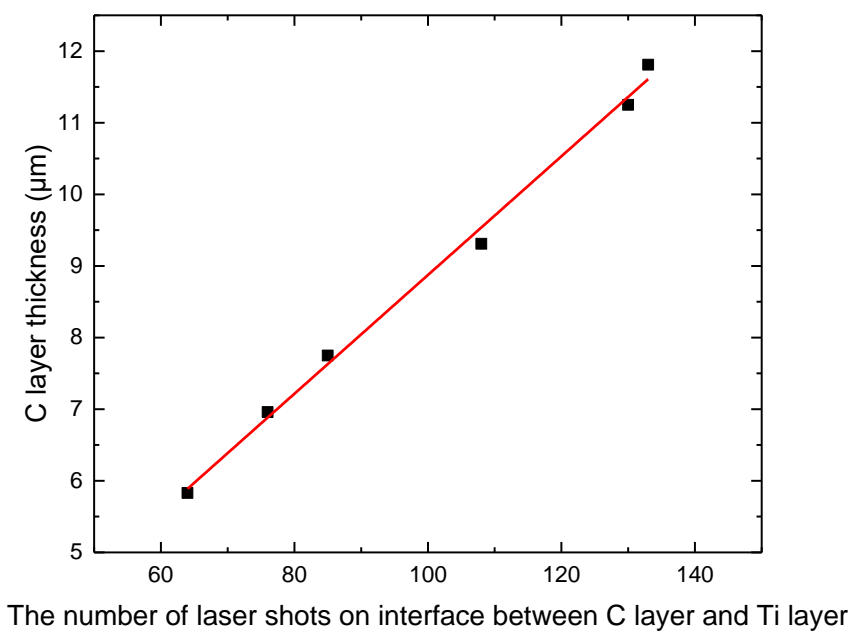

Fig. 6. Correlation of the number of laser shots reach on the interface between the $\mathrm{C}$ layer and the $\mathrm{Ti}$ layer and the $C$ layer thickness which is measured by FIB. The ablation rate of $(82.9 \pm 0.9) \mathrm{nm} / \mathrm{shot}$ can be achieved.

\section{$3.4 \mathrm{H}$ content of deposition layer on the screw surface}

The $\mathrm{H}$ content measurement is very important for the study of fuel retention in fusion devices. The depth profiles of the $\mathrm{H}$ content on the surface of different screws are shown in fig. 7 . The LIBS intensity ratio of $\mathrm{H}$ and $\mathrm{C}$ are measured to reduce the influence of laser energy fluctuation. The $\mathrm{H}$ intensities on the surface of exposed screws are higher than on the surface of the new screw without plasma exposure. From the third laser shot, the intensity ratios of $\mathrm{H}$ and $\mathrm{C}$ are almost same for all screws. To achieve the quantitative H content, LIA-QMS signals are calibrated by a calibration leak with known flow rate. The Detail to this calibration procedure can be found in [23]. The depth profiles of $\mathrm{H}(\mathrm{m} / \mathrm{z}=2 \mathrm{u} / \mathrm{e})$ content from the LIA-QMS are shown in fig. 7. The $\mathrm{H}$ contents of approximately $1.1 \times 10^{21}$ atoms $/ \mathrm{m}^{2}$ on the exposed screw are higher than the $\mathrm{H}$ contents of approximately $3.5 \times 10^{20}$ atoms $/ \mathrm{m}^{2}$ on the new screws. The LIA-QMS results are in agreement with the LIBS results. On the unexposed screws, the H mainly came from the $\mathrm{H}_{2} \mathrm{O}$ adsorption from the air. Before the screws exposed on the stellarator plasma in W7$\mathrm{X}$, the PFCs were baked more than $100{ }^{\circ} \mathrm{C}$ to reduce the impurities such as $\mathrm{H}_{2} \mathrm{O}$. The SEM photos on the surface of the new screw and exposed screw \#14 are shown in fig. 8. Compared to the new screw surface, the deposition layer is found locally on the surface of exposed screws during the plasma exposure in the $\mathrm{W} 7-\mathrm{X}$, because the screws were recessed to the surrounding baffle graphite surface and located in net deposition zones. The a-C:H layer is rich in $\mathrm{H}$ and results in that the LIBS and LIA-QMS 
intensities of $\mathrm{H}$ on the surface of the exposed screw is higher. In addition, the $\mathrm{O}$ content is below the limit of detection, thus not too much $\mathrm{O}$ is co-deposited at this location.

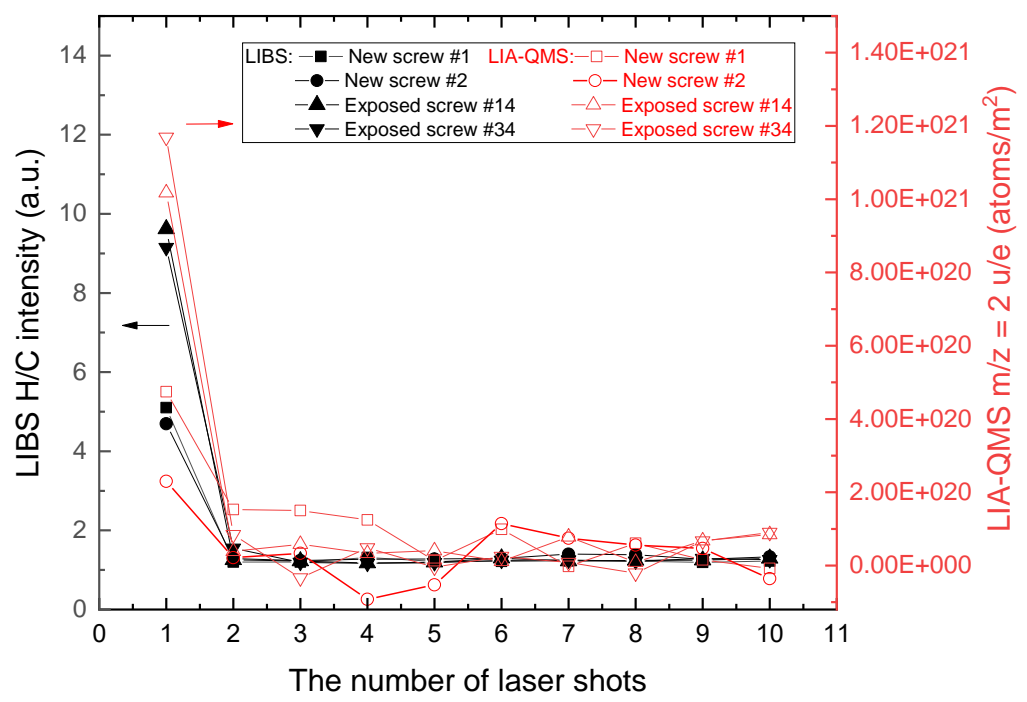

Fig. 7. The depth profiles of the H content of LIBS and LIA-QMS on the surface of the new screws and exposed screws.
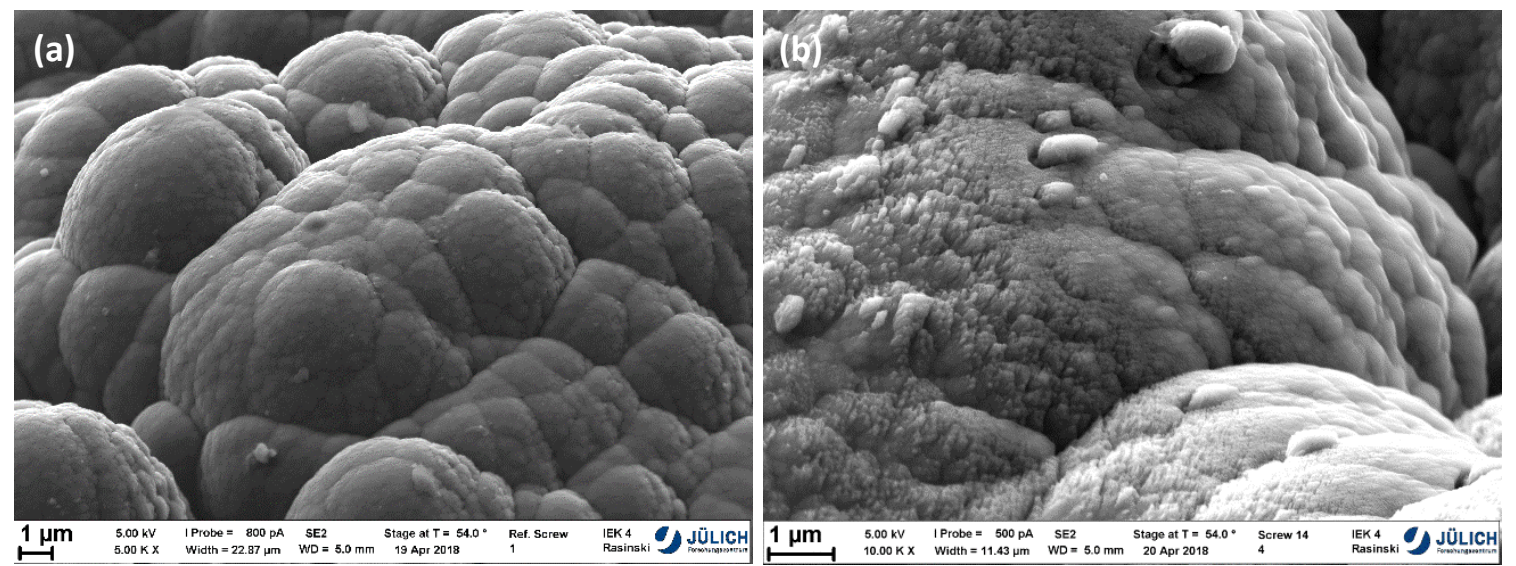

Fig. 8. The SEM photos on (a) the surface of the new screw without plasma exposure and (b) the surface of the exposed screw \#14. 


\section{Conclusion}

The ps-LIBS method is employed to investigate the elemental depth distribution of screws from the divertor baffle of W7-X in OP1.2a. The screws have a multilayer structure with a certain thickness of C layer from $5.8 \mu \mathrm{m}$ to $12.0 \mu \mathrm{m}$ on the top surface. This structure provides a unique sample to determine the erosion and deposition as well as fuel retention on C layer. LIBS depth analysis clearly shows the different behaviors of $\mathrm{H}, \mathrm{C}, \mathrm{Ti}$ and Mo intensities with depth. The $\mathrm{H}$ on the surface has very strong intensity due to the surface adsorption. From the third laser shot, the $\mathrm{C}$ depth profile and the $\mathrm{H}$ depth profile are consistent. The Ti intensities from the interface layer are increases and then decreases with depth. The Mo intensity from the TZM substrate increases and then plateaus with depth. The LIA-QMS results about $\mathrm{C}$ depth profile are in agreement with LIBS results.

The linear correlation approach has been successfully applied for improving the elemental depth profiles and identifying the interface between the $\mathrm{C}$ layer and the Ti layer. The results of the FIB thickness and the LIBS are well fitted and result in that the LIBS ablation rate of $(82.9 \pm 0.9) \mathrm{nm} / \mathrm{shot}$ can be achieved by a laser energy density of $6.5 \mathrm{~J} / \mathrm{cm}^{2}$. The erosion and deposition are able to be obtained comparing the thicknesses which are measured before and after the plasma exposure. In addition, LIBS intensities from $\mathrm{H}$ retention on the surface of the screws which were exposed to stellarator discharge plasma in OP1.2a are higher than on the surface of the new screw without plasma exposure. The $\mathrm{H}$ contents which are achieved quantitatively by LIA-QMS on exposed screws and new screws are approximately $1.1 \times 10^{21}$ atoms $/ \mathrm{m}^{2}$ and approximately $3.5 \times 10^{20}$ atoms $/ \mathrm{m}^{2}$, respectively. The SEM result shows that the high $\mathrm{H}$ intensity is due to the plasma deposited a-C:H layer which is found locally on the surface of exposed screws. These indicate that the two exposed screws were located at the net deposition zones at the inboard and outboard side baffles. The studies well demonstrate the ability for the thickness and $\mathrm{H}$ retention measurement by depth profile analysis of LIBS on the W7-X divertor baffle screws. This would help us to develop in situ LIBS system to monitor the elemental distribution for the 3D PWI study on the divertor of W7-X in further operation phases.

\section{Acknowledgments}

This work was supported by the National Science Foundation of China (No. 11605023), the National Key R\&D Program of China (No. 2017YFE0301300), China Postdoctoral Science Foundation (Nos. 2017T100172, 2016M591423). This work has been carried out within the framework of the EUROfusion Consortium and has received funding from the Euratom research and training programme 2014-2018 under grant agreement No 633053. The views and opinions expressed herein do not necessarily reflect those of the European Commission. 


\section{References}

[1] H.-S. Bosch, et al., Final integration, commissioning and start of the Wendelstein 7-X stellarator operation, Nucl. Fusion 57 (2017) 116015.

[2] J. Geiger, et al., Physics in the magnetic configuration space of W7-X, Plasma Phys. Control. Fusion 57 (2015) 014004.

[3] Y. Liang, et al., Diagnostic set-up and modelling for investigation of synergy between 3D edge physics and plasma-wall interactions on Wendelstein 7-X, Nucl. Fusion 57 (2017) 066049.

[4] S. Brezinsek, Plasma-Surface Interaction and Plasma-Edge Studies in Wendelstein 7-X with Passively Cooled Graphite Divertor, Presented at EPS 45th Conference on Plasma Physics 2018 (Prague).

[5] K. Sugiyama, et al., Ion beam analysis of H and D retention in the near surface layers of JT-60U plasma facing wall tiles, J. Nucl. Mater. 363-365 (2007) 949-954.

[6] M. Mayer, et al. Carbon erosion and deposition on the ASDEX Upgrade divertor tiles, J. Nucl. Mater. 337-339 (2005) 119-123.

[7] T. Shibahara, et al. Hydrogen retention of JT-60 open divertor tiles exposed to HH discharges, Nucl. Fusion 46 (2006) 841-847.

[8] M. Zlobinski, et al., Laser induced desorption as tritium retention diagnostic method in ITER, Fusion Eng. Des. 86 (2011) 1332-1335.

[9] V. Philipps, et al., Development of laser-based techniques for in situ characterization of the first wall in ITER and future fusion devices, Nucl. Fusion 53 (2013) 093002.

[10] C. Li, et al., Review of LIBS application in nuclear fusion technology, Front. Phys. 11 (2016) 114214.

[11] R. Hai, et al., Laser-induced breakdown spectroscopic characterization of impurity deposition on the first wall of a magnetic confined fusion device: Experimental Advanced Superconducting Tokamak, Spectrochim. Acta Part B 87 (2013) 147-152.

[12] C. Li, et al., Characterization of deuterium retention and co-deposition of fuel with lithium on the divertor tile of EAST using laser induced breakdown spectroscopy, J. Nucl. Mater. 463 (2015) 915-918. [13] A. Semerok, et al., Laser induced breakdown spectroscopy application in joint European torus, Spectrochim. Acta Part B 123 (2016) 121-128.

[14] J. Karhunen, et al., Applicability of LIBS for in situ monitoring of deposition and retention on the ITER-like wall of JET - comparison to SIMS, J. Nucl. Mater. 463 (2015) 931-935.

[15] P. Paris, et al., Development of laser induced breakdown spectroscopy for studying erosion, deposition, and fuel retention in ASDEX upgrade, Fusion Eng. Des. 98-99 (2015) 1349-1352. 
[16] M. Suchonova, et al., Determination of deuterium depth profiles in fusion-relevant wall materials by nanosecond LIBS, Nuclear Mater. Energy, 12 (2017) 611-616.

[17] D. Zhao, et al., Remote in situ laser-induced breakdown spectroscopic approach for diagnosis of the plasma facing components on experimental advanced superconducting tokamak, Rev. Sci. Instrum. 89 (2018) 073501.

[18] Q. Xiao, et al., In-situ analysis of the first wall by laser-induced breakdown spectroscopy in the TEXTOR tokamak: Dependence on the magnetic field strength, J. Nucl. Mater. 463 (2015) 911-914.

[19] C. Li, et al., Laser-induced breakdown spectroscopy for Wendelstein 7-X stellarator limiter tile analysis, Phys. Scr., T170 (2017) 014004.

[20] J. Oelmann, et al., Depth resolved analysis of hydrogen in W7-X graphite components using laserinduced ablation-quadrupole mass spectrometry (LIA-QMS), Nucl. Mater. Energy 18 (2019) 153-158. [21] O. Neubauer, et al., Diagnostic setup for investigation of plasma wall interactions at Wendelstein 7-X, Fusion. Eng. Des. 96-97 (2015) 891-894.

[22] S. Brezinsek et al., Fuel retention studies with the ITER-Like Wall in JET, Nucl. Fusion 53 (2013) 083023.

[23] J. Oelmann, et al., Depth-resolved sample composition analysis using laser-induced ablationquadrupole mass spectrometry and laser-induced breakdown spectroscopy, Spectrochim. Acta Part B 144 (2018) 38-45.

[24] C.P. Dhard, et al., Preparation of erosion and deposition investigations on plasma facing components in Wendelstein 7-X, Phys. Scr T170 (2017) 014010.

[25] B.N. Chichkov, et al., Femtosecond, picosecond and nanosecond laser ablation of solids, Appl. Phys. A 63 (1996) 109-115.

[26] J. Anzano, et al., Laser-induced plasma spectroscopy for plastic identification, Polym. Eng. Sci. 40 (2000) 2423-2429.

[27] G. Galbacs, et al., Semi-quantitative analysis of binary alloys using laser-induced breakdown spectroscopy and a new calibration approach based on linear correlation, Spectrochim. Acta Part B 56 (2001) 1159-1173. 